\title{
Frankreichs Suche nach einem europäischen Narrativ
}

\author{
Olivier Rozenberg*
}

In regelmäßigen Abständen vermitteln die Wahlen zum Europäischen Parlament den Eindruck, dass das Verhältnis der Französischen Republik zu Europa problematisch ist. 1992 wurde der Vertrag von Maastricht mit knapper Mehrheit befürwortet. 1999 zeigte sich fast jeder zweite gewählte französische Europaabgeordnete den europäischen Verträgen gegenüber abgeneigt. 2005 setzte das ,Non“ der Franzosen beim Referendum dem Vertrag über eine Verfassung für Europa (Verfassungsvertrag) ein Ende. 2014 ist schließlich der Front National, eine rechtsextreme Partei, welche den Euro ablehnt, Sieger der Europawahl, gemessen sowohl an der Anzahl der französischen Stimmen als auch der Frankreich zustehenden Sitze im Europäischen Parlament. In Anbetracht der historischen Schlüsselrolle, welche Frankreich beim Fortgang der europäischen Integration seit den 1950er Jahren gespielt hat, ${ }^{1}$ überrascht diese Reihe von Ereignissen. Diese Häufung von Krisen und Spannungen seit über 20 Jahren lässt vermuten, dass sie nicht nur die Ablehnung der Exekutive oder die Sorgen der Bevölkerung in Anbetracht der wirtschaftlichen Schwierigkeiten des Landes ausdrücken. Vielmehr ist davon auszugehen, dass diese Skepsis gegenüber Europa tiefer liegende Ursachen hat und unmittelbar mit dem französischen Verständnis von Europa zusammenhängt. Die französischen Eliten haben ein öffentliches Narrativ zur Begründung der europäischen Integration geformt, das mit der Formel ,Europa der Macht‘ (,Europe puissance') zusammengefasst werden könnte. Im Folgenden wird der Inhalt dieses Narrativs dargestellt und verdeutlicht, inwieweit es sich abgenutzt hat, bevor die Schwierigkeiten thematisiert werden, das bestehende durch ein neues Narrativ zu ersetzen.

\section{Das irreführende Narrativ über das starke Europa}

„L'euro fait la force‘ (,mit dem Euro sind wir stark'): Dieser von der französischen Regierung Ende der 1990er Jahre verwendete Slogan, um bei den Bürgern für die Einführung des Euro zu werben, ist ein gutes Beispiel für das Narrativ ,Europa der Macht‘. Er postuliert, dass die europäische Integration für Frankreich Macht, Stärke und Effizienz mit sich bringt. ${ }^{2}$ Der Machtzuwachs durch Europa entstehe nicht in erster Linie durch Skaleneffekte, welche die Integration erzeugt, sondern durch die geografische Dimension Europas. Die Überlegung ist im Grunde eher geopolitischer denn wirtschaftlicher Natur. Während des Kalten Kriegs wurde die Europäische Wirtschaftsgemeinschaft (EWG) als eine Einheit wahrgenommen, welche der Sowjetunion und, in geringerem Maße, den USA die Stirn bieten konnte. Seit den 1990er Jahren wird die Europäische Union als ein Schutz gegen

* Olivier Rozenberg, Associate Professor, Centre d'études européennes, Sciences Po, Paris.

1 Cornelia Woll/Richard Balme: France. Between Integration and National Sovereignty, in: Simon Bulmer/ Christian Lequesne (Hrsg.): The Member States of the European Union, 1. Auflage, Oxford 2005, S. 97-118; Craig Parsons: A Certain Idea of Europe, Ithaca 2003.

2 Gérard Bossuat: La France et la construction de l'unité européenne, de 1919 à nos jours, Paris 2012; Helen Drake (Hrsg.): French Relations with the European Union, Abingdon 2005; Marion Gaillard: Politique européenne de la France de 1950 à nos jours, Brüssel 2010. 
die Konkurrenz von Niedriglohnländern und multinationalen Konzernen im Rahmen der Globalisierung dargestellt.

Die Idee eines starken Europas ist mit der nüchternen Erkenntnis verbunden, dass der internationale Einfluss Frankreichs abnimmt. Die militärische Niederlage von 1940, die kritische Situation der Nachkriegszeit und die Dekolonisation haben den politischen und administrativen Eliten bewusst gemacht, dass das Land nicht mehr den Anspruch erheben kann, alleine den Gang der Ereignisse in der Welt zu beeinflussen. Europa bietet demzufolge sowohl die Möglichkeit des Verzichts als auch die einer Utopie: Verzicht auf einen gewissen messianischen Anspruch der Nation, welcher von der französischen Revolution geerbt wurde, und zugleich Utopie, diesen Anspruch auf europäischer Ebene aufrechtzuerhalten. Das Narrativ beinhaltet implizit die Idee, dass Frankreich mit und durch Europa das erreichen will, wozu es alleine nicht mehr in der Lage ist. Ihm wohnt somit eine kongeniale Doppeldeutigkeit inne. Die europäische Integration und die Souveränitätsübertragungen, die sie beinhaltet, werden nur insofern akzeptiert, als sie zur Wiederherstellung oder zur Beibehaltung französischer Macht beitragen. Europa ist somit im Grunde genommen ein Mittel zum Zweck der nationalen Belange.

Unzählige Beispiele belegen die Doppeldeutigkeit von pro-europäischen Positionierungen Frankreichs seit den Anfängen der europäischen Integration. In ihrer euroskeptischen Variante kann beispielsweise die ambivalente Positionierung unter der Präsidentschaft Charles de Gaulles (1958-1969) hervorgehoben werden, welcher zwar bereit war, die Römischen Verträge einzuhalten, gleichzeitig aber mit seiner ,Politik des leeren Stuhls` das Recht eines jeden Staates verteidigte, seine vitalen Interessen geltend zu machen. Ein Beispiel für die europafreundlichere Version ist François Mitterrand (1981-1995), der in seiner Regierungspolitik ab 1983 seinen ursprünglichen radikal-sozialistischen Politikentwurf durch das Projekt eines vereinigten Europas ersetzte, gleichzeitig aber eine stark intergouvernementale Vision im institutionellen Bereich verteidigte. So setzte er während der Verhandlungen zum Vertrag von Maastricht die Säulenkonstruktion des europäischen ,Bauwerks' durch, welche die gemeinsame Außenpolitik vor jeglicher Vergemeinschaftung bewahrte. In einer pragmatischen Version lässt sich schließlich feststellen, dass Jacques Chirac (1995-2007) sich bemühte, Frankreich auf die Einführung des Euro vorzubereiten, um sich anschließend aus den Zwängen des Stabilitäts- und Wachstumspakts zu befreien. ${ }^{3}$ In Frankreich selbst lassen sich ebenfalls Spuren dieser Mehrdeutigkeit des Narrativs ,Europa der Macht' finden: Ein Beispiel ist das Zögern einer Reihe von französischen Institutionen, die Konsequenzen der Verträge für das nationale Recht und das Regierungs- und Verwaltungshandeln zu akzeptieren. ${ }^{4}$ So dauerte es bis 1989 , bis der Staatsrat (Conseil d'État) den Vorrang des europäischen vor dem nationalen Recht vollständig anerkannte. Regierungen und Parlamentarier waren noch bis 2005 darin einig, dass das Prinzip des Souveränitätstransfers nicht in die französische Verfassung aufgenommen, sondern hierüber von Fall zu Fall entschieden werden solle. In den 1990er und 2000er Jahren verabschiedeten Abgeordnete und Senatoren bei verschiedenen Gelegenheiten Gesetze, welche dem europäischen Recht widersprachen, wie etwa jene über die Vogeljagd, die konträr zu einer europäischen Richtlinie aus dem Jahre 1979 sind.

3 Hussein Kassim: France and the European Union under the Chirac Presidency, in: Alistair Cole/Patrick Le Galès/Jonah Levy (Hrsg.): Developments in French Politics, 4. Auflage, New York 2008, S. 258-276.

4 Olivier Rozenberg: France: Genuine Europeanisation or Monnet for Nothing?, in: Simon Bulmer/Christian Lequesne (Hrsg.): The Member States of the European Union, 2. Auflage, Oxford 2013, S. 57-84; Emiliano Grossman (Hrsg.): France and the EU. After the Referendum, Journal of European Public Policy 7/2007. 


\section{Die langsame Abnutzung des europäischen Narrativs}

Europa wurde in Frankreich als Bedingung für den nationalen Machterhalt legitimiert. Dieses Narrativ ist bis heute nicht verschwunden. So ließ sich beobachten, dass die französische Ratspräsidentschaft 2008 von Nicolas Sarkozy auf eine Weise vorbereitet, durchgeführt und zelebriert wurde, als ob sie allein seinem persönlichen und dem Ruhme Frankreichs dienen würde. ${ }^{5}$ Die Ergebnisse des Referendums über den Verfassungsvertrag und jene der Europawahl im Jahr 2014 weisen jedoch darauf hin, dass sich die Stärke dieses Narrativs seit mehr als zwei Jahrzehnten abnutzt. Meinungsumfragen, welche die Beliebtheit der Europäischen Union messen, bestätigen diesen relativen Verfall: Während 1973 nur fünf Prozent der Franzosen die Zugehörigkeit zur EWG als eine schlechte Sache betrachteten, waren dies im Jahre 2010 bereits 20 Prozent. ${ }^{6}$ Die Wirtschaftskrise seit 2008 hat diesen Euroskeptizismus innerhalb der Bevölkerung noch verstärkt. Grafik 1 zeigt das wachsende Misstrauen der Franzosen gegenüber der Europäischen Union. Dieses hat in sieben Jahren um mehr als 23 Prozentpunkte zugenommen. Allerdings ist dieser Befund in seiner Bedeutung in doppelter Hinsicht zu relativieren: Einerseits zeigt sich diese Tendenz nicht nur in Frankreich, sondern auch im Durchschnitt der öffentlichen Meinung in den Mitgliedstaaten der ,EU-27`. Andererseits ist das Misstrauen gegenüber der nationalen Regierung in Frankreich - abgesehen von der Zeit unmittelbar nach einer Wahl - ausgeprägter als jenes gegenüber der Europäischen Union.

\section{Abbildung 1: Die Entwicklung des Vertrauens in die Europäische Union und in die französische Regierung (2007-2013)}

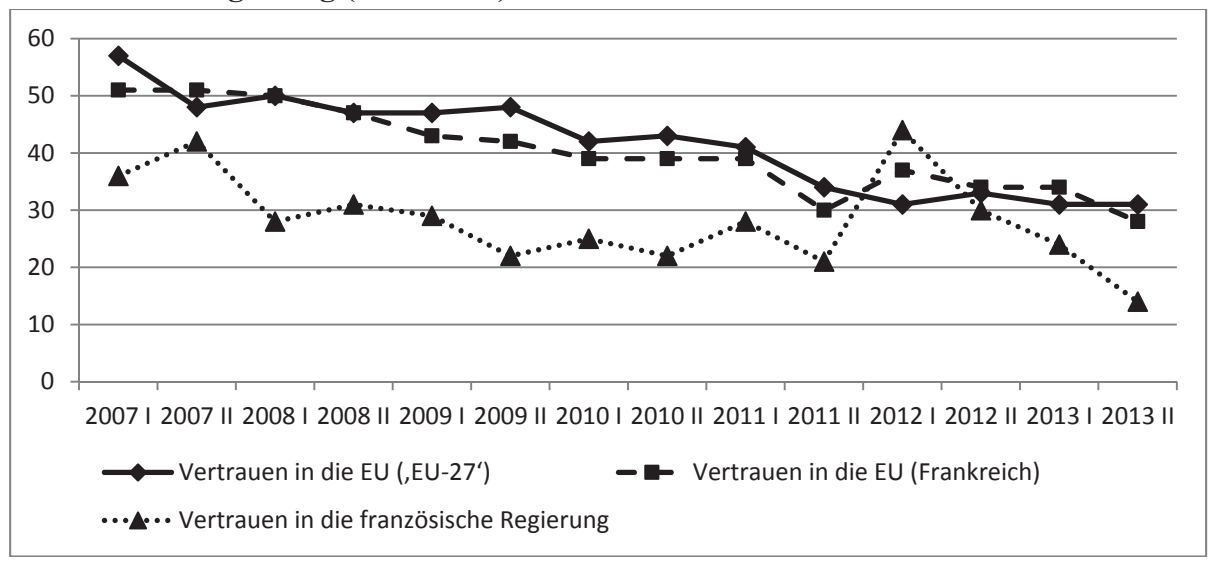

Quelle: Eigene Darstellung auf Basis der Daten aus Europäische Kommission: Standard Eurobarometer, abrufbar unter: http://ec.europa.eu/public_opinion/archives/eb_arch_en.htm (letzter Zugriff: 2.10.2014).

Trotz dieser doppelten Relativierung scheint es, dass der Euroskeptizismus in der französischen Gesellschaft wächst. Das Narrativ ,Europa der Macht‘ ist immer weniger in der

5 Christian Lequesne/Olivier Rozenberg: The French Presidency of 2008: the Unexpected Agenda, Stockholm 2008; Helen Drake: France and the European Union, in: Alistair Cole/Sophie Meunier/Vincent Tiberj (Hrsg.): Developments in French Politics, New York 2013, S. 218-232.

6 Europäische Kommission: Standard Eurobarometer, abrufbar unter: http://ec.europa.eu/public_opinion/archives /eb_arch_en.htm (letzter Zugriff: 2.10.2014). 
Lage, die Bürger für Europa zu gewinnen. Die politischen Parteien und Entscheidungsträger melden ebenfalls unterschiedliche Vorbehalte gegenüber der europäischen Integration und ihren Entscheidungsverfahren an. Die Kritik von Seiten der politischen Parteien an den beiden Extremen des politischen Spektrums ist seit Langem ein Mittel zur wirksamen Abgrenzung gegenüber den Regierungsparteien. Die relative Kontinuität in der europäischen Politik durch die linken und rechten Regierungen wird sowohl vom Front National als auch von der kommunistischen Bewegung als abgekartetes Spiel kritisiert. Europa ist besonders für den Front National und seine Präsidentin, Marine Le Pen, Tochter des schillernden Begründers der Partei, nützlich. Seit 2011 verfolgt die Partei eine Strategie der Normalisierung, mit der sie sich bemüht, die umstrittensten Aspekte ihres Verständnisses von der französischen Identität vergessen zu machen. Da der Front National durch diese Normalisierungsstrategie Gefahr läuft, sich immer weniger von den anderen Parteien zu unterscheiden, ermöglicht das Thema Europa der Partei, gegenüber den Wählern eine Radikalität geltend zu machen, die besser gesellschaftlich akzeptabel, da frei von Rassismus, ist. Die Kritik an Europa hat dabei jedoch nichts anekdotisches, bildete sie 2012 den Wählerbefragungen zufolge doch eine der wichtigsten Motivationen für die Wahl von Le Pen. ${ }^{7}$ Allerdings ist anzumerken, dass diese Normalisierungsstrategie ihre Grenzen im Europäischen Parlament findet. Le Pen gelang es nicht, eine Fraktion im Europäischen Parlament zu bilden, sie konnte nicht die dazu notwendige Anzahl an Abgeordneten aus genügend Mitgliedstaaten zusammen bringen. Neben den extremistischen Parteien gibt es in Frankreich ,issue parties', die sich um europäische Themen gebildet haben. Sie hatten in den 1990er und 2000er Jahren einen gewissen Erfolg, bevor sie politisch bedeutungslos wurden: Hierzu gehören auch die Souveränisten, welche hauptsächlich aus dem konservativen Lager hervorgegangen sind, sowie eine ländliche Gruppierung, die sich ursprünglich zur Verteidigung des Jagdrechts gebildet hatte.

Die Europakritik eint nicht nur die extremistischen Parteien, sondern das Thema entzweit auch regelmäßig die Regierungsparteien, rechts wie links. Der Spaltung der Konservativen in den 1990er Jahren zwischen Anhängern des Vertrags von Maastricht und den Verfechtern der nationalen Souveränität folgte jene der Linken zwischen Befürwortern des Verfassungsvertrags und Antiliberalen in den 2000er Jahren. Diese Spaltungen zeigen, dass in Frankreich das Lager der Unterstützer Europas nicht auf eine der beiden politischen Familien reduziert werden kann. Die französische Rechte ist zerrissen zwischen ihren gaullistischen Ursprüngen, die die nationale Unabhängigkeit betonen, sowie einem liberalen Flügel, der sich umso mehr mit Europa identifiziert, je besser dies die Möglichkeit bietet, einen eigenen Parteiflügel aufrechtzuerhalten. Ein Teil der Regierungslinken protestierte energisch gegen die liberale Logik der Europäischen Union. Darüber hinaus sind diese unterschiedlichen ideologischen Spannungen regelmäßig ein Spielball persönlicher politischer Erwägungen. Von der Attacke Chiracs auf den damaligen französischen Präsident Valéry Giscard d'Estaing 1979 bis hin zu Parlamentspräsident Claude Bartolone, der 2013 den Premierminister zu einer ,Konfrontation“ mit Deutschland aufrief, kann beobachtet werden, dass die Infragestellung der Verträge, von Allianzen oder europäischen Politiken eine bewusst gewählte Strategie innenpolitischer Herausforderer ist, um die Führung in ihrem jeweiligen Lager zu beanspruchen. Diese Taktik ist nicht ohne Risiken, könnte sie doch das staatsmännische Bild der Herausforderer schädigen. Dennoch

7 Nona Mayer: From Jean-Marie to Marine Le Pen. Electoral Change on the Far Right, in: Parliamentary Affairs 1/2013, S. 160-178. 
wird sie regelmäßig eingesetzt, da sie es sowohl ermöglicht, der Führungsperson des eigenen Lagers Unfähigkeit bei der Verteidigung nationaler Interessen vorzuwerfen als auch sich vom gegnerischen Lager abzugrenzen. Zudem zeigen einige erfolgreiche Karrieren europakritischer Politiker, dass das Risiko, das eigene Bild als Staatsmann langfristig zu beschädigen, begrenzt ist. Im konservativen Lager wurde François Fillon, ehemals Gegner des Vertrags von Maastricht, 2007 Premierminister. Im linken Lager wurde Laurent Fabius, Gallionsfigur der Gegner des Verfassungsvertrags, fünf Jahre später Außenminister.

\section{Die Wirklichkeitsferne des Narrativs ,Europa der Macht‘}

Was dem Beobachter der europapolitischen Debatte in Frankreich auffällt, ist nicht so sehr der in Zyklen auftretende kritische Diskurs über die europäische Integration, der bereits die französische öffentliche Debatte in den 1950er Jahren durch die virulente und gleichzeitige Kritik von kommunistischer wie gaullistischer Seite kennzeichnet. Seit der Präsidentschaft Mitterrands ist das Bemerkenswerteste vielmehr die Schwierigkeit pro-europäischer Entscheidungsträger, ihre Position in der öffentlichen Debatte zu artikulieren und zu verteidigen. Analysiert man die Programme und Reden der Regierungsparteien, so wird die halbherzige Unterstützung der Integration in ihren Modalitäten und manchmal sogar in ihrem Prinzip selbst augenscheinlich. So wurde auf der politischen Rechten bereitwillig betont, dass die Europäische Union mit ihrem Reglementierungs- und Vereinheitlichungsanspruch aufhören muss. Bei den Linken befürwortet der Parti socialiste (PS) seit Langem eine soziale Umorientierung der europäischen Integration. Während der Kampagne für die Präsidentschaftswahlen 2012 bekam die Infragestellung der gemeinsamen Politiken durch pro-europäische Kandidaten sowohl hinsichtlich ihres Ausmaßes als auch hinsichtlich ihrer Resonanz eine neue Dimension. ${ }^{8}$ Der scheidende Präsident Sarkozy machte den Verbleib Frankreichs im Schengen-Raum von einer tiefgreifenden Erneuerung des Systems abhängig. Sein sozialistischer Herausforderer Hollande kündigte an, dass er den Stabilitätspakt in der vorgelegten Fassung nicht unterschreiben werde - wozu er sich dann schließlich doch entschloss. Zum offenen und identitätsbezogenen Euroskeptizismus der extremistischen Parteien kommt somit eine, weiche Form des Euroskeptizismus‘ der Entscheidungsträger in den Regierungsparteien hinzu.

Eine solche Schwierigkeit, sich öffentlich zur europäischen Integration zu bekennen, ist wahrscheinlich der Notwendigkeit geschuldet, die Euroskeptiker innerhalb des jeweiligen politischen Lagers für sich zu gewinnen. Sie ist aber auch ein Zeichen, so scheint es, für die Abnutzung des großen nationalen Narrativs ,Europa der Macht' zur Begründung der Integration $^{9}$. Dieser Verschleiß rührt einerseits daher, dass verschiedene Präsidenten, insbesondere Giscard d'Estaing und Mitterrand, nicht damit gegeizt haben, mit diesem europäischen Narrativ ihre Politik zu rechtfertigen. Der Rückgriff auf europapolitische Argumente ermöglichte es Giscard d'Estaing, sich gekonnt vom Erbe der gaullistischen Präsidenten abzugrenzen. Für Mitterrand bot sich mit Europa eine Ersatzutopie für die neomarxistische Politik an. Andererseits, und weitaus negativer, haben die Mitterrand folgenden französischen Präsidenten und Premierminister ,Brüssel` die Verantwortung für unpo-

8 Renaud Dehousse/Angela Tacea: The French 2012 Presidential Election. A Europeanised Contest, in: Les Cahiers européens de Sciences Po 2/2012; Helen Drake: Everywhere and Nowhere: Europe and the World in the French 2012 Elections, in: Parliamentary Affairs 1/2013, S. 124-141.

9 Vivien A. Schmidt: Trapped by their ideas. French élites' discourses of European integration and globalization, in: Journal of European Public Policy 7/2007, S. 992-1009. 
puläre politische Entscheidungen angelastet. Beispielsweise nahm Chirac kurz nach seiner Wahl 1995 seine Wahlversprechen zurück und begründete dies mit der notwendigen Vorbereitung Frankreichs auf den Euro. Das Narrativ ,Europa der Macht' hat darunter gelitten, zu oft genutzt worden zu sein, sowohl positiv als auch negativ. Darüber hinaus scheint dieses Narrativ heute aus zwei weiteren Gründen ausgedient zu haben.

Das Narrativ ,Europa der Macht“ ist zunächst ein Instrument der Legitimation. Die europäische Integration lässt sich damit begründen, dass sie in der Lage ist, einen Machtzuwachs mit sich zu bringen und somit politische Ergebnisse zu erzielen. Offenkundig bringt diese Art der Begründung Europas aber Probleme mit sich, wenn die Ergebnisse ausbleiben, was sowohl in wirtschaftlicher als auch geopolitischer Hinsicht der Fall ist. Das schwache Wachstum der französischen Wirtschaft relativiert ebenso wie die Schwierigkeit, Sozialdumping zu unterbinden oder Finanzspekulationen zu besteuern, die Idee, dass Europa stark macht. Geopolitisch untergraben die unterschiedlichen strategischen Ausrichtungen der Mitgliedstaaten wie etwa zu Beginn des Irakkriegs 2003 oder Frankreichs Isolierung bei seinen Interventionen in Afrika, ebenfalls die Idee eines Europas als Machtverstärker. Die Unterstützung Europas leidet daran, dass es an konkreten politischen Ergebnissen mangelt. Dieser Befund scheint selbstverständlich zu sein. Doch ist es wichtig festzustellen, dass Europa umso mehr darunter leidet, je mehr seine Daseinsberechtigung in Frankreich, aber auch in anderen Ländern wie beispielsweise dem Vereinigten Königreich, nur mit bestimmten funktionalen oder praktischen Zwecken verknüpft wird. Nationale Narrative, die etwa die (Wieder-)Einbindung des Staates, die Modernisierung der Gesellschaft oder die Anbindung an den Westen in den Vordergrund stellen, sind wahrscheinlich weniger von diesen Schwierigkeiten betroffen.

Zweitens ist das Narrativ ,Europa der Macht', wie bereits erwähnt, auch eine irreführende Begründung der europäischen Einigung, weil das zentrale Anliegen der Vertreter dieses Narrativs die Verteidigung der Größe Frankreichs ist. Konkret ist die europäische Integration in den Augen der Franzosen nicht Opfer seiner Misserfolge, sondern seiner Erfolge. Trotz der Schwächen der Europäischen Union hat sie sich seit den 1980er Jahren vertieft. Dies belegen die Vielzahl der verabschiedeten europäischen Rechtsakte, der Übergang zur Entscheidung mit qualifizierter Mehrheit im Rat und die Europäisierung von Politikbereichen, welche zuvor von einer Vergemeinschaftung ausgenommen waren (soziale Sicherungssysteme, Bildungssystem, Verteidigung etc.). Die Europäisierung der nationalen Politik ist offenkundig. Sie steht nicht unbedingt im Widerspruch zur Idee ,Europa der Macht‘ im Sinne Frankreichs. Die nationalen politischen Eliten verweisen auf die, übrigens fälschliche, Vorhersage Jacques Delors, wonach 80 Prozent der wirtschaftlichen und sozialen Gesetzgebung aus Europa kommen werde. Wenn die Vertiefung der Integration allerdings den Handlungsspielraum französischer Politiker einschränkt, kommt die Realitätsferne des Mythos' eines französischen Europas zum Vorschein. Seit mehreren Jahren gibt es zahlreiche Beispiele europäischer Entscheidungen hierfür, sei es das Verbot oder die Rückerstattung staatlicher Beihilfen für Unternehmen, die Kontrolle von Firmenfusionen oder das Ende von Staatsmonopolen für zahlreiche ehemals öffentliche Dienstleistungen. 2005 wurde Frankreich zum ersten Mal wegen Nichtanwendung europäischen Rechts zu einer Geldbuße verurteilt. Besonders im Rahmen der Wirtschafts-, Finanz- und Eurozonenkrise seit 2009 hat die Europäische Union ein in der Theorie strikteres System zur Kontrolle der nationalen Haushaltsdefizite umgesetzt. Die europäischen Zwänge wurden so zu einem zentralen Element der nationalen öffentlichen Debatten und beispielsweise von der Opposition genutzt, um der seit 2012 regierenden Sozialistischen Partei wirt- 
schaftliche Inkompetenz vorzuwerfen. Doch auch der französische Präsident Hollande selbst weiß dieses Argument zu nutzen, wenn er mit „Brüssel“ die Gesamtheit seiner großen innenpolitischen Entscheidungen rechtfertigt, von Steuererhöhungen bis zum Austausch des Premierministers.

Vom Bild eines starken Europa, welches das verlorene Prestige Frankreichs wiederherzustellen vermag, ging man so unmerklich zu jenem eines sanktionierenden Europas über, welches die Umsetzung schmerzhafter innerer Reformen fordert. Die Erweiterung der Europäischen Union und die zunächst nur wirtschaftliche, dann aber auch politische Dominanz Deutschlands untergruben die Glaubwürdigkeit des Gedankens eines französischen Europas vollends. Die Erweiterungsrunde des Jahres 2004, die im Parlament und in den Medien wenig debattiert wurde, ist von den politischen und administrativen Eliten als ein Verzicht auf eine weitere Vertiefung der europäischen Integration empfunden worden. Die Nostalgie eines Europas der zwölf Mitgliedstaaten drückte sich indirekt in der Idee einer Union für das Mittelmeer aus, welche von Sarkozy im Rahmen der französischen Ratspräsidentschaft 2008 vorgestellt wurde. Sein ursprüngliches Konzept schloss Deutschland nicht mit ein, das so auf seine östliche Einflusssphäre verwiesen werden sollte, während sich Frankreich eine Führungsrolle in der südlichen Zone der europäischen Nachbarschaft sichern wollte. So unterschiedlich der Kontext auch sein mag, so verweist die Sehnsucht nach einer Union der Länder des ,Club Med' gegenüber dem in haushaltspolitischen Fragen als streng wahrgenommenen Deutschland, welches während der Präsidentschaft Hollandes regelmäßig auch so beschrieben wird, ebenfalls auf die Idee, eine neue regionale Integration zu schaffen, in der Frankreich sein verlorenes Prestige wiederfinden könnte.

\section{Die schwierige Erneuerung des europäischen Narrativs in Frankreich}

Die Erosion des Narrativs ,Europa der Macht` hängt somit teilweise mit dessen Struktur zusammen. In diesem Kontext scheint es den französischen politischen Entscheidungsträgern an Vorstellungskraft zu mangeln, um einen Ersatzmythos zu entwerfen. So ist beispielsweise festzustellen, dass es ihnen schwer fällt, den großen Reden und Projektideen ihres engen Partners Deutschland, sei es das Kerneuropa-Projekt von Wolfgang Schäuble und Karl Lamers von $1994^{10}$ oder die Rede des damaligen deutschen Außenministers Joschka Fischer im Jahr 2000 an der Humboldt-Universität, ${ }^{11}$ etwas entgegenzusetzen. Die französischen Präsidenten sind regelmäßig dazu angehalten, über Europa zu reden, doch sind diese Deklarationen, die oft den Eindruck erwecken, nur widerwillig nach einer mehrwöchigen Kampagne der Zeitung Le Monde formuliert worden zu sein, für alle konsensfähig, um nicht zu sagen platt, und selten bindend, um nicht zu sagen nutzlos. So formulierte Präsident Hollande im Mai 2013 während einer Pressekonferenz sein europäisches Projekt mit vier Prioritäten: eine Wirtschaftsregierung der Eurozone mit einem Präsidenten an ihrer Spitze, einen europäischen Plan gegen die Arbeitslosigkeit, den Ausbau eines Europas der Energie und die Einleitung einer gemeinsamen Investitionsstrategie. Es ist festzustellen, dass er hier Ideen übernahm, welche, insbesondere im Falle der Wirtschaftsregierung, bereits vor langer Zeit formuliert worden waren. Durch die Schwierigkeiten Frankreichs, seine Verpflichtungen in Haushaltsfragen einzuhalten, wurde die in der Rede vor-

10 Wolfgang Schäuble/Karl Lamers: Überlegungen zur europäischen Politik, 1.9.1994, abrufbar unter: https:// www.cducsu.de/upload/schaeublelamers94.pdf (letzter Zugriff: 1.10.2014).

11 Joschka Fischer: Vom Staatenverbund zur Föderation - Gedanken über die Finalität der europäischen Integration, in: integration 3/2000, S. 149-156. 
gebrachte Priorität der Wirtschaftsregierung jedoch wieder verworfen. Einige Monate später, als die Europäische Kommission präzise Reformempfehlungen für Frankreich vorlegte, die sowohl den Arbeitsmarkt als auch das Rentensystem betrafen, tat Präsident Hollande seine Ablehnung öffentlich kund und stritt der Kommission das Recht ab, sich in die inneren Angelegenheiten seines Landes einzumischen. In Anbetracht der seitdem von ihm umgesetzten Reformen, welche den von der Kommission geforderten sehr ähnlich sind, kommt die ablehnende Äußerung Hollandes einer Verleugnung gleich.

Abgesehen von den Mehrdeutigkeiten des Projekts der Wirtschaftsregierung erstaunt bei dieser Rede - wie auch bei den Reden von Hollandes Amtsvorgänger Sarkozy - das Fehlen einer großen europäischen Vision, so als ob die Aneinanderreihung von konkreten Prioritäten die Problematik der Neuformulierung eines französischen Narrativs über Europa kaschieren könnte. Diese Schwierigkeit hängt vermutlich mit der nationalen politischen Kultur einerseits und dem französischen politischen System andererseits zusammen.

Es ist immer schwierig, von politischer Kultur zu sprechen, schließlich könnten sich Vorurteile an die Stelle der empirischen Analyse schleichen. Außerdem kann die Analyse der politischen Kultur widersprüchliche Ergebnisse liefern: Einige Autoren hoben hervor, dass die französische politische Kultur des Exzeptionalismus die pro-europäische Wende der Sozialisten in den 1980er Jahren genährt habe ${ }^{12}$. Ungeachtet der Gefahren einer solchen kulturellen Analyse scheint es, dass sich die von den politischen und administrativen Eliten Frankreichs geteilten Normen mehr und mehr von Europa in seiner jetzigen Form entfernt haben, und dies unter zwei Aspekten: ein Vorbehalt gegenüber der freien Marktwirtschaft einerseits und eine Verbeugung vor dem politischen Voluntarismus ${ }^{13}$ andererseits. Hinsichtlich des ersten Aspekts zeigen vergleichende Meinungsumfragen, dass die Franzosen - Akademiker eingeschlossen - gemeinsam mit den anderen südeuropäischen Ländern den Prinzipien des Wirtschaftsliberalismus' am skeptischsten gegenüberstehen. ${ }^{14}$ Gehörten die Franzosen in den gleichen Umfragen vor mehr als zehn Jahren noch zu den Europafreundlichsten, so stellt die heutige Zurückhaltung gegenüber dem freien Markt eine nicht minder bedeutsame Hürde für Frankreichs Verhältnis zu Europa dar. Christian Lequesne hat gezeigt, dass die Vorbehalte gegenüber der Erweiterung nicht nur auf der Angst gründen, dass Frankreich seine zentrale Position verlieren könnte. ${ }^{15}$ Durch die wiederholte Forderung, dass Europa mehr sein müsse als nur ein großer Markt, offenbaren die politischen Verantwortlichen ihre geringe Zustimmung zum Binnenmarktprojekt.

Zweitens idealisiert die französische politische Kultur den Begriff des politischen Voluntarismus. Diese Idealisierung wird von zahlreichen Autoren konstatiert. Sie wird erst in der Langzeitperspektive verständlich und verweist, jenseits der Französischen Revolution, auf die vom Staat ausgehende Bildung der französischen Nation. Das Primat des Politischen richtet sich auf die Institutionen und neigt dazu, die Personalisierung und Zentralisierung der kollektiven Regierungsführung und dem Mehrebenensystem vorzuziehen. Daraus ergibt sich eine Problemdiagnose, welche gleichermaßen von den politischen Verantwortlichen des linken wie des rechten Parteienspektrums vorgebracht wird: Es fehle eine Führungsperson, welche imstande ist, Europa eine politische Richtung vorzugeben. In die-

12 Martin Marcussen et al.: Constructing Europe? The evolution of French, British and German state identities, in: Journal of European Public Policy 4/1999, S. 614-633.

13 Damit ist die Fähigkeit der Politik gemeint, die Gesellschaft zu verändern.

14 Luc Rouban: La France en Europe, in: Pascal Perrineau/Luc Rouban (Hrsg.): La politique en France et en Europe, Paris 2007, S. 409-424.

15 Christian Lequesne: La France dans la nouvelle Europe, Paris 2008. 
sem Sinne entstand der Europäische Rat auf Initiative der Franzosen und wird der Ära Delors mit Nostalgie gedacht. Die Schaffung des Amtes eines ständigen Präsidenten des Europäischen Rates durch den Vertrag von Lissabon wurde begrüßt, während andere Strategien der Legitimierung zurückhaltend aufgenommen wurden (Parlamentarisierung, Transparenz, Konstitutionalisierung, ,better regulation“ etc.). Die Forderung nach einem solchermaßen ,politischen Europa ‘ wird regelmäßig mit einer derartigen Vehemenz vorgetragen, dass das Konzept polymorph, um nicht zu sagen sinnentleert geworden ist. ${ }^{16}$

Es geht hier nicht darum, über die Berechtigung der Marktwirtschaft oder des politischen Voluntarismus zu entscheiden, sondern hervorzuheben, dass die Werte, welche die französischen Entscheidungsträger teilen, eher dazu führen, sie von Europa zu entfernen und es ihnen somit schwer machen, eine neue Begründung für Europa zu formulieren. Die Politiker und leitenden Beamten ähneln darin übrigens den von Justine Lacroix untersuchten französischen Intellektuellen, welche der Europäischen Union vorwerfen, es fehle ihr an Gestalt, also an einer wahrhaftigen politischen Gemeinschaft, und an einem Kopf, welcher eine kohärente politische Richtung vorgeben könnte. ${ }^{17}$

Ein weiterer Argumentationsstrang, warum es so schwierig ist, das französische Narrativ der europäischen Integration zu erneuern, betrifft die Institutionen der V. Republik. Der präsidentielle Zentralismus bietet ein wirksames Instrument, die großen nationalen Prioritäten in der Europapolitik zu definieren und zu verfolgen. Er behindert jedoch die Ausarbeitung einer kohärenten Vision für Europa aus zwei Gründen. Erstens zwingt die Präsidentschaftswahl mit zwei Wahlgängen die Kandidaten der zwei großen Parteien Union pour un Mouvement Populaire (UMP) und PS - dazu, einen europapolitischen Diskurs zu führen, der innerhalb eines jeden Lagers sowohl die Europhilen als auch die Euroskeptiker überzeugt. Dieser Ansatz der großen Regierungsparteien, praktisch jeden einzubinden, findet sich zwar in den meisten heutigen Demokratien. In Frankreich betrifft er jedoch durch das Zusammenwirken des Ernennungsverfahrens des Präsidenten ${ }^{18}$ und der Aufspaltung der jeweiligen Wählerschaft entlang des Themas die Europapolitik in besonderer Weise. Die europapolitischen Positionen der Kandidaten der PS und der UMP ähneln somit sorgfältig abgewogenen Parteitagsbeschlüssen. 2007 kündigte Sarkozy an, nach dem Scheitern des Verfassungsvertrags einen neuen Vertrag aushandeln zu wollen und diesen nicht einem Referendum zu unterziehen, thematisierte öffentlich aber hauptsächlich seine ablehnende Haltung gegenüber einem Beitritt der Türkei. ${ }^{19} 2012$ versah der damalige Präsidentschaftskandidat Hollande seine Absicht, den Fiskalpakt neu zu verhandeln, mit einer ambitionierten europäischen Agenda, welche die Schaffung von Eurobonds, einer europäischen Ratingagentur und eine Richtlinie zum Schutz der öffentlichen Da-

16 Helen Drake: France in Europe, Europe in France. The Politics of Exceptionalism and their Limits, in: Tony Chafer/Emmanuel God (Hrsg.): The End of the French Exception? Decline and Revival of the 'French Model', Basingstoke 2010, S. 187-202.

17 Diese Intellektuellen würden laut Lacroix schließlich eine imaginäre europäische Doxa ersinnen, in welcher die Vergangenheit von der Schuld des Westens geprägt und die Nation als Kriegsverursacherin verstanden wird. Vgl. Justine Lacroix: Une Europe sans corps ni tête. La pensée française après le 29 mai, in: Justine Lacroix/Ramona Coman (Hrsg.): Les résistances à l'Europe, Brüssel 2007, S. 155-165; siehe auch: Justine Lacroix: La pensée française à l'épreuve de l'Europe, Paris 2008.

18 Im Gegensatz zu Präsidentschaftswahlen oder Wahlen mit zwei Wahlgängen tragen Parlamentswahlen und/ oder die Wahlen mit einem Wahlgang eher dazu bei, dass jede politische Gruppierung ihre Eigenarten und Unterschiede zu anderen hervorheben kann, ohne konsensfähig sein zu müssen, auch wenn dies vor oder nach den Wahlen zur Verabschiedung eines Koalitionsabkommens führt.

19 Renaud Dehousse: Nicolas Sarkozy l'Européen, in: Jacques de Maillard/Yves Surel (Hrsg.): Les politiques publiques sous Sarkozy, Paris 2012, S. 153-188. 
seinsvorsorge vorsah, dies alles abgestützt mit einem europäischen Budget „im Dienste der großen Zukunftsprojekte“ ${ }^{\text {20 }}$. Die Wahlanalyse zeigt, dass er in beiden Wahlgängen, in gleichem Maße von Befürwortern und Gegnern des Verfassungsvertrags 2005 unterstützt wurde. ${ }^{21}$ Die ,Synthese à la hollandaise ${ }^{6}$ zum Thema Europa war somit ein Schlüssel zu seinem Erfolg.

Ein weiterer institutioneller Faktor ist die Vormachtstellung des Präsidenten in der Europapolitik Frankreichs, die ihn nicht dazu verpflichtet, sich an sein Wahlprogramm zu halten. ${ }^{22}$ Der Erfolg Hollandes 2012 ist hier ein frappierendes Beispiel. Dem Versprechen, den Fiskalpakt neu zu verhandeln, folgten keine wirklichen Taten obwohl Letzteres, wie bereits erwähnt, der Grundstein für den politischen Erfolg des sozialistischen Kandidaten war. Der Präsident hat den Pakt stattdessen im Austausch für symbolische Gegenleistungen akzeptiert, welche im Nachhinein als marginal betrachtet werden können. Trotz einigen Unmutes fand die Ratifizierung im Herbst 2012 ohne große Probleme statt. ${ }^{23}$ Es ist hier weniger von Bedeutung, ob der französische Präsident anders hätte agieren können, vielmehr ist relevant, dass das parlamentarische Verfahren keine Hürde darstellt. Der Kandidat konnte ein Programm definieren, von dem er wusste, dass es ihn nur geringfügig verpflichten würde. Eine solche Diskrepanz zwischen den Wahlversprechen und der Regierungspolitik begünstigt nicht das Erstellen von kohärenten Programmen und Grundsätzen durch die Regierungsparteien. Dies gilt nicht nur für die Europapolitik. Die europäische Vision des Kandidaten wird meistens eilig am Vorabend von Wahlkampfveranstaltungen mit Europabezug erarbeitet. Die Schwierigkeit der französischen Eliten, Europa zu denken, erklärt sich somit teilweise, aber nur teilweise, durch die Oberflächlichkeit der programmatischen Arbeit der Parteien. Diese Oberflächlichkeit wird durch die Vorrangstellung des Präsidenten im politischen System begünstigt.

\section{Fazit: eine euroskeptische Wende der französischen Eliten?}

Dieser Beitrag hat versucht zu erklären, warum die politisch Verantwortlichen in Frankreich solche Schwierigkeiten haben, die europäische Integration mit einem Narrativ zu versehen. Das alte Narrativ ,Europa der Macht‘ hat nach und nach mit dem Abstieg Frankreichs auf den zweiten Platz in der Europäischen Union an Kraft verloren, ohne dass sich, aus Gründen, die sowohl mit der politischen Kultur der Eliten als auch mit den Institutionen der V. Republik zusammenhängen, ein anderes Narrativ zur Begründung der europäischen Einigung durchsetzen konnte. Das Bemerkenswerteste dabei ist, dass diese narrative Lücke die Durchführung der europäischen Politik Frankreichs nicht wirklich behindert hat. Das Land hat, in gewisser Weise, die Mittel, eine Strategie zu verfolgen, welche im

20 Vgl. François Hollande: Mes soixante engagements pour la France (26.1.2012), abrufbar unter: http://www. parti-socialiste.fr/articles/les-60-engagements-pour-la-france-le-projet-de-francois-hollande (letzter Zugriff: 11.8.2014); Christian Lequesne: A new President in the Elysée: Continuity and Change in French EU Politics, in: Journal of Common Market Studies, Annual Review 1/2013, S. 42-54.

21 Jérôme Jaffré: La victoire étroite de François Hollande, in: Pascal Perrineau (Hrsg.): Le vote normal. Les élections présidentielle et législatives d'avril-mai-juin 2012, Paris 2013, S. 133-160.

22 Die politische Macht des Präsidenten ist an die Parlamentsmehrheit gekoppelt. Doch seit 2002, zum Teil aufgrund des Übergangs der Dauer der Präsidentschaft von sieben auf fünf Jahre, verfügten die drei aufeinanderfolgenden Präsidenten über eine parlamentarische Fraktion, welche in der Assemblée Nationale alleine eine Mehrheit bildete.

23 Anja Thomas/Angela Tacea: The French Parliament and the EU - 'shadow control' through the government majority, in: Claudia Hefftler/Christine Neuhold/Olivier Rozenberg/Julie Smith (Hrsg.): Palgrave Handbook of National Parliaments and the European Union, Basingstoke im Erscheinen. 
öffentlichen Raum nicht wirklich thematisiert und begründet wird. Die institutionelle Stärke des Präsidenten und die Konstanz einer pro-europäischen leitenden Beamtenschaft waren bis jetzt ausreichend, um dies auszugleichen. Derzeit ist jedoch zu beobachten, dass Frankreich Schwierigkeiten hat, seinen Platz in Europa zu finden. Dies verleitet die Exekutive regelmäßig dazu, nach dem Vorbild Chiracs, eher das Prestige als das nationale Interesse zu verteidigen. So akzeptierte er 2000, dass die Parität zwischen Frankreich und Deutschland bei der Sitzverteilung im Europäischen Parlament aufgehoben wurde, nur um diese bei der Stimmengewichtung im Rat besser verteidigen zu können.

Ist die Kluft zwischen der pro-europäischen Politik der französischen Regierungen und einem öffentlichen Raum, in dem die Kritik an Europa dominiert, langfristig haltbar? Die langsame programmatische Entwicklung der Regierungsparteien, die hier die Freizügigkeit der Personen rückgängig machen wollen, dort das Verbot der staatlichen Beihilfen kritisieren, beziehungsweise eine Renationalisierung der Umweltpolitik predigen, lässt daran zweifeln. Im Mai 2013 hat die UMP in ihrem Programm festgeschrieben, dass kein europäischer Rechtsakt anzunehmen sei, ohne dass ein anderer im gleichen Politikbereich abgeschafft wird.

Die Lehre Antonio Gramscis behält, auch wenn sie etwas abgedroschen klingt, ihre Relevanz für die französische Europapolitik: Die Schlacht der Ideen verliert man nicht ungestraft. Mit gewissem Abstand ermisst man, dass der Vertrag von Maastricht durch einen Diskurs von Mitterrand antizipiert wurde, in dem er Europa zum Horizont seiner Politik und der Nation machte. So gesehen scheint der weiche Euroskeptizismus der Regierungsparteien eine kritische Entwicklung der Europapolitik Frankreichs einzuleiten.

Übersetzt von Jéronimo Barbin. 